\title{
Evaluation of a novel powered vascular stapler in laparoscopic nephrectomy
}

Viraj A Master ${ }^{1}$, Steve Leung ${ }^{2}$, Toby Page ${ }^{3}$, Anthony Blacker ${ }^{4}$, Simon Williams ${ }^{5}$, Aniruddha Chakravarti ${ }^{6}$, Grenville Oades ${ }^{7}$, Gurminder Mann $^{8}$, Chandru Sundaram ${ }^{9}$, Alberto Breda ${ }^{10}$, Carlos Hernandez ${ }^{11}$, Erin E Creedon ${ }^{12}$, Michael L Schwiers ${ }^{12}$, David W Singleton ${ }^{12}$, Jason R Waggoner $^{12}$ and Elliott J Fegelman ${ }^{12}$

${ }^{1}$ Emory University, Atlanta, GA, USA

${ }^{2}$ Western General Hospital, Edinburgh, UK

${ }^{3}$ Freeman Hospital, Newcastle-Upon-Tyne, UK

${ }^{4}$ University Hospitals Coventry and Warwickshire, Coventry, UK

${ }^{5}$ Royal Derby Hospital, Derby, UK

${ }^{6}$ New Cross Hospital, Wolverhampton, UK

${ }^{7}$ Queen Elizabeth University Hospital, Glasgow, UK

${ }^{8}$ Nottingham Hospitals, Nottingham, UK

'Indiana University, Indianapolis, IN, USA

${ }^{10}$ Fundacio Puigvert, Barcelona, Spain

${ }^{11}$ Gregorio Maranon, Madrid, Spain

${ }^{12}$ Ethicon Endo-Surgery, Inc., Johnson \& Johnson, Cincinnati, OH, USA

\begin{abstract}
Objective: The purpose of this study was to determine if use of a powered vascular stapler (PVS) during laparoscopic nephrectomy or nephroureterectomy procedures would yield an equivalent rate of additional hemostatic interventions to Standard of Care (SOC) staplers.

Methods: A prospective, randomized, multicentre, controlled study was conducted comparing the use of PVS to SOC in laparoscopic nephrectomy or nephroureterectomy. The primary performance endpoint was the incidence of additional intraoperative haemostatic interventions, and the primary safety endpoint was the frequency of postoperative bleeding-related interventions. Equivalence was denoted by a $95 \%$ confidence interval for the difference in intervention rates between PVC and SOC with a limitation of $3 \%$

Results: There were 136 subjects in the SOC group and 130 subjects in the PVS group who completed the study. The rates of intraoperative haemostatic interventions were $13.6 \%$ and $18.4 \%$ for SOC and PVS groups, respectively, $(\mathrm{p}=0.107)$. The upper bound of the difference in intervention rates exceeded the $3 \%$ criterion for equivalence. Postoperative bleeding that required intervention was experienced in two subjects (1.4\%) in the SOC group and one (0.8\%) in PVS. There were four adverse events rated as serious for SOC and none for PVS. Estimated blood loss was similar between the two groups.
\end{abstract}

Conclusions: The PVS performed safely and effectively in nephrectomy and exhibited a rate of haemostatic intervention that was not statistically different than the SOC.

\section{Introduction}

Complete simple nephrectomy or radical nephrectomy may be performed for a variety of conditions that cause irreversible kidney damage such as chronic infection, obstruction, serious trauma, calculus disease, and tumours $[1,2]$. The most common kidney cancer (renal cell carcinoma) is managed with open, laparoscopic, or robotic nephrectomy, while laparoscopic nephroureterectomy is typically employed for urothelial carcinoma affecting the upper urinary tract [3]. Nephrectomy has also been an option for treatment of renovascular hypertension due to non-correctable renal artery disease in children [4]. In kidney transplantations, laparoscopic living donor nephrectomy has become the predominant standard of care for renal allograft procurement at many institutions [5-7].

With the evolution of minimally invasive techniques, there is an increased need for more specialized surgical instrumentation in urologic procedures. To achieve haemostasis in laparoscopic nephrectomy or nephroureterectomy, stapling may be considered advantageous compared to surgical clips since a stapler allows for division and ligation of an artery or vein in a single firing. Moreover, when a stapling device misfires during surgery it may be immediately observed, and bleeding issues addressed intraoperatively. Complications such as bleeding or damage to nearby organs may occur during nephrectomy

${ }^{\star}$ Correspondence to: David Singleton, Ethicon Endo-Surgery, Inc., Johnson \& Johnson, Cincinnati, OH, USA, Tel: +1 (513) 337-1027; E-mail: dsingl12@its.jnj.com Key words: nephrectomy, powered stapler, haemostasis, renal cell carcinoma

Received: October 30, 2019; Accepted: November 11, 2019; Published: November 15, 2019 
procedures $[8,9]$. The emergence of powered staplers provides an additional level of control allowing for less unwanted movement, more precise placement of staples, and potential for reduced risk of procedure related complications $[10,11]$.

A narrow-profile articulating powered vascular stapler (PVS) was developed that displayed enhanced haemostasis compared to larger conventional staplers in thoracic procedures [12]. Importantly, this stapler tip has a slight angulation that allows for easier placement around structures. It is also smaller in size, allowing for less dissection of hilar vessels to gain enough space for vessel ligation. Four staggered rows of staples (two on either side of the cut line) are produced by the PVS, as opposed to the typical six rows (three on each side of the cut line) for SOC staplers. With use of this powered vascular stapler, the risk of bleeding and/or damage to nearby organs may be reduced, due to the decreased size and greater articulation of the device. The objective of the present study was to assess the frequency of haemostatic interventions required following transection of the renal artery (RA) and renal vein (RV) with the PVS, or the surgeon's standard of care stapler, during laparoscopic nephrectomy or nephroureterectomy.

\section{Methods}

\section{Study design}

This was a prospective, randomized, controlled, multicentre study utilizing two centres in the United States, seven United Kingdom institutions, and two Spanish centres. The protocol and consent documents were approved by each investigator's Institutional Review Board (IRB) or Independent Ethics Committee (IEC). The study was conducted in accordance with Good Clinical Practice (GCP) and the Declaration of Helsinki, as well as any other applicable local, state and federal regulatory requirements, and is registered with ClinicalTrials.gov (NCT02807376). The test device was the ECHELON FLEX $^{\mathrm{m}}$ Powered Vascular Stapler (PVS, Ethicon Endo-Surgery, Inc., Cincinnati $\mathrm{OH}$ ) compared to the standard of care stapler (SOC) used at each institution. The principal investigators at each site were surgeons qualified by education, experience, and training to perform the procedures. Surgeons who lacked previous training with the PVS device, were required to complete a minimum of two procedures using PVS, prior to randomizing their first subject.

\section{Selection of subjects}

Subjects who were scheduled to undergo a simple or radical laparoscopic nephrectomy or nephroureterectomy were considered for study participation. Additional inclusion criteria were: performance status of 0-1 (Eastern Cooperative Oncology Group classification) if applicable; American Society of Anaesthesiologists (ASA) physical status score $\leq 3$; no prior history of partial or wedge nephrectomy on the affected kidney; willingness to consent and comply with studyrelated evaluation and treatment schedule and; at least 18 years of age. Exclusion criteria were any of the following: pregnancy; chemotherapy or radiation within 30 days prior to the procedure; physical or psychological condition which would impair study participation or; unsuitable for study participation determined by the Investigator. Subjects who were randomized and in whom the study procedure began (first incision) were considered enrolled and were included in analyses of performance and safety. Subjects were randomized to the surgeons' current SOC stapler or PVS in a 1:1 ratio. Randomization could occur at any time prior to surgery up to transection of the vessel(s).

\section{Surgical treatments}

Each subject underwent a simple laparoscopic or radical nephrectomy or a laparoscopic nephroureterectomy in compliance with the standard procedure at the respective study site. The principal investigator at each site performed the transection of the RA/RV with either the SOC stapler or the PVS with $35 \mathrm{~mm}$ vascular white reloads (Ethicon Endo-Surgery, Inc., Cincinnati, OH). Subjects continued with their current medical care while in the study, including medications. Anticoagulants and NSAIDS taken preoperatively up to 30 days prior to the procedure and the stop dates were recorded, as were any medications prescribed due to adverse events (AEs). The protocol included four visits: screening, surgery, postoperative through discharge, and a postoperative visit/phone call (approximately 4 weeks after surgery or as per SOC).

\section{Performance and safety variables}

The primary performance measure was the incidence of haemostatic interventions or procedures completed for intraoperative bleeding related to transection of the RA and RV following use of SOC stapler or PVS. Interventions included: additional stapling, over-sewing, clip placement, compression, use of suture, sealant, buttress, energy device, transfusion of blood or blood products, or an additional surgical procedure (e.g., conversion to open). No haemostasis intervention was defined as no bleeding at the staple line or bleeding that stopped after initial blotting of staple line. The primary safety variable was the number and percentage of subjects requiring postoperative haemostatic interventions that were considered related to transection of RA/RV (e.g., need for blood product transfusion or additional surgical procedure following postoperative bleeding). All AEs were recorded through the duration of the study, regardless of the relationship to the procedure or study device. In addition to the primary measures, surgeons were asked to complete a device satisfaction survey and a Surgery Task Load Index (Surg-TLX). Six specific components of the index were measured: levels of mental fatigue, physical fatigue, hurried pace, procedure complexity, surgeon stress, and distraction in the operating environment. Each component was scored on a 0 to 100 scale, and an overall score was calculated as the average of the six components.

\section{Statistical analysis}

Summary statistics and $95 \%$ confidence intervals were determined for the number and frequency of interventions for the set of SOC and PVS subjects separately. This was performed based on the total number of firings. It was expected to have at least 300 firings in each group, with a sample size of approximately 250 total subjects. This was considered adequate for descriptive summarization of the number and frequency of haemostasis interventions. To establish that the intervention rate was not increased compared to SOC, a 95\% confidence interval for the difference in proportion of firings requiring intervention for PVS minus SOC was calculated and PVS was considered to have acceptable performance if the upper bound of the one-sided 95\% confidence interval did not exceed 3\%. A direct comparison of the rates of intervention between groups was performed using the Chi-square test $\left(\chi^{2}\right)$.

The number and percentage of subjects requiring haemostasis interventions postoperatively that were classified as related to RA/ $\mathrm{RV}$ transection (e.g., need for blood product transfusion or additional surgical procedure) were summarized for each group as a primary safety endpoint. Safety was also assessed through the incidence of device and procedure-related AEs and serious adverse events (SAEs), 
which were coded using the Medical Dictionary for Regulatory Activities (MedDRA). Analysis of performance endpoints was based on the full analysis set, which included all randomized subjects who had the procedure performed and provided data on the number of surgical interventions. Analysis of safety endpoints was based on the safety set, which included all randomized subjects on whom the procedure was started.

\section{Results}

\section{Subject selection and demographic data}

A total of 302 subjects were screened for study participation, and 270 were randomized to undergo laparoscopic nephrectomy or nephroureterectomy using either SOC stapler $(n=139)$ or PVS $(\mathrm{n}=131)$ for transection of RA and RV (Figure 1). Subjects who completed the study through the 4-week postoperative visit, included $136(97.8 \%)$ in the SOC group and $130(99.2 \%)$ in the PVS group. Three discontinuations in the SOC group were due to surgical reasons (specifically conversion to open) per study protocol. One of the PVS group was lost to follow-up after successful completion of the surgery and discharge from the hospital. All discontinued subjects were included in both the effectiveness and safety analyses as they contributed data on stapler firings and assessment for haemostasis interventions. Table 1 summarizes key demographic information and baseline characteristics of the full analysis set, which included subjects randomized at 11 centres. Mean age of the subjects was 63.8 years (range 19 to 94), and a majority $(87.4 \%)$ were White. No meaningful differences were observed between treatment groups for any characteristics including the distribution of ASA physical status classification.

\section{Surgical characteristics}

Table 2 describes the types of surgical procedures performed, location of affected kidneys, and surgical characteristics for the full analysis set. The procedures included 221 (81.9\%) nephrectomies and 49 (18.1\%) nephroureterectomies. Most of the affected kidneys were unilateral, except for three subjects in whom bilateral kidney pathology was noted. The two device groups showed comparable results for estimated intraoperative blood loss and mean operative duration. Blood transfusions were slightly higher in the SOC group [3 subjects (2.2\%) for SOC compared to 1 subject $(0.8 \%)$ for PVS]. Three SOC subjects were converted to an open procedure compared to zero in the PVS group. One of these was converted due to left renal vein bleeding that was considered possibly related to the use of the SOC stapler.

\section{Intraoperative interventions}

Intraoperative haemostatic interventions performed on the RA or $\mathrm{RV}$ are summarized in table 3 for the full analysis set. No meaningful differences were observed in the distributions of types of vessel transections between groups. In the SOC subjects, $13.6 \%$ (42 of 309) of vessel transections required haemostatic intervention compared to $18.4 \%$ (56 of 305 ) in the PVS group. The two rates of intervention did not differ statistically $(p=0.107)$. However, the difference in intervention rates between groups (PVS minus SOC) was $4.8 \%$, and the upper bound of the one-sided $95 \%$ confidence interval was $9.6 \%$, which exceeded the pre-defined 3\% criterion for similar performance. The higher haemostatic intervention rate observed in the PVS group was driven by a greater utilization of clips ( 44 vs 31 ). The numbers of other types of intervention were similar between SOC and PVS groups. Transfusions and/or additional surgical procedures were the most serious category of intraoperative haemostatic intervention. One SOC subject required intraoperative blood product transfusion and conversion to open surgery due to left renal vein bleeding.

\section{Postoperative interventions}

Two SOC subjects (1.4\%) and $1(0.8 \%)$ in the PVS group experienced an occurrence of postoperative bleeding that required intervention (Table 4). In the SOC group, one subject received a blood transfusion and an additional surgical procedure involving laparoscopic evacuation of hematoma and application of haemostatic product. A postoperative haemorrhage $\mathrm{AE}$ was recorded that was considered related to the study procedure and possibly to the study device. The other SOC subject required a transfusion for which an $\mathrm{AE}$

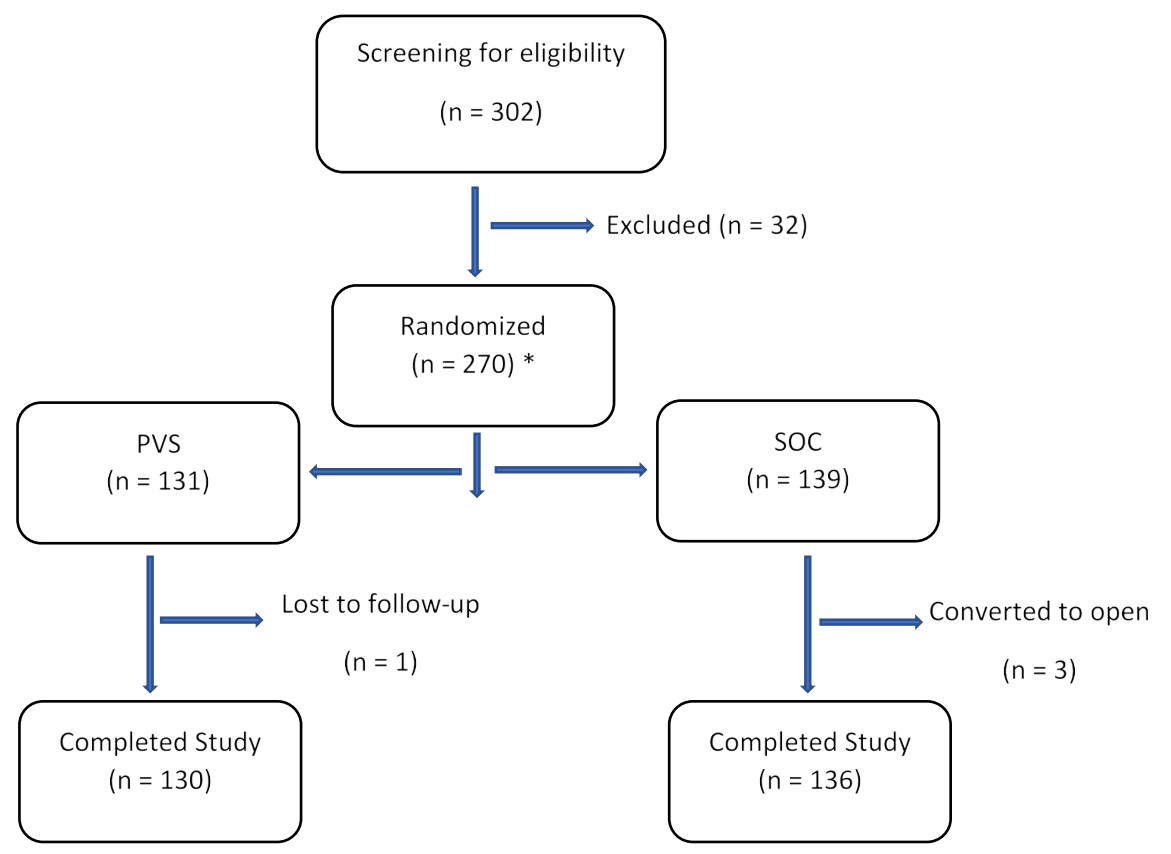

Figure 1. Participant Flow Diagram. *All 270 subjects who underwent surgery were included in effectiveness and safety analysis sets. 
of "postoperative bleeding" was identified as related to the procedure but of unknown relationship to the stapler device. In the PVS group, one subject received a postoperative blood transfusion due to a drop of haemoglobin level, which was recorded as an AE with unknown relationship to the PVS device.

\section{Adverse events}

Overall the frequency of AEs and SAEs were similar between groups and representative of the types of events that would be expected in these types of procedures. No subjects in either group reported AEs that resulted in discontinuation from the study. In the SOC group, 4 subjects (2.9\%) reported stapler device-related AEs (two post-procedural haemorrhages, one haematuria, and one hematoma); 3 subjects (2.3\%) had device-related AEs in the PVS group (one procedural haemorrhage, and two decreased haemoglobin). One or more procedure-related AEs occurred in 46 (33.1\%) subjects in the SOC group and $45(34.4 \%)$ in the PVS group, with no meaningful differences in the types of reported events between the groups. These

Table 1. Subject Demographic Characteristics. *American Society of Anesthesiologists (ASA) physical status classification

\begin{tabular}{|l|c|c|c|}
\hline Variable & $\begin{array}{c}\text { Standard of Care } \\
\mathrm{N}=139\end{array}$ & $\begin{array}{c}\text { Powered Vascular } \\
\text { Stapler N=131 }\end{array}$ & $\begin{array}{c}\text { Total } \\
\mathrm{N}=270\end{array}$ \\
\hline Race n (\%) & $119(85.6 \%)$ & $116(89.2 \%)$ & $235(87.4 \%)$ \\
\hline White & $13(9.4 \%)$ & $11(8.5 \%)$ & $24(8.9 \%)$ \\
\hline $\begin{array}{c}\text { Black or African } \\
\text { American }\end{array}$ & $2(1.4 \%)$ & $2(1.5 \%)$ & $4(1.5 \%)$ \\
\hline Asian & $5(3.6 \%)$ & $1(0.8 \%)$ & $6(2.2 \%)$ \\
\hline Other & $64.1(14.0)$ & $63.6(13.8)$ & $63.8(13.9)$ \\
\hline Age (years) & $67.0(23.0-94.0)$ & $66.0(19.0-88.0)$ & $66.5(19.0-94.0)$ \\
\hline Mean (SD) & $71(51.1 \%)$ & $71(54.2 \%)$ & $142(52.6 \%)$ \\
\hline Median (range) & $68(48.9 \%)$ & $60(45.8 \%)$ & $128(47.4 \%)$ \\
\hline Gender, n (\%) & & & $32(11.9 \%)$ \\
\hline Male & $16(11.5 \%)$ & $16(12.2 \%)$ & $144(53.3 \%)$ \\
\hline Female & $72(51.8 \%)$ & $72(55.0 \%)$ & $94(34.8 \%)$ \\
\hline ASA* Physical Status & $51(36.7 \%)$ & $43(32.8 \%)$ & \\
\hline ASA I & &
\end{tabular}

Table 2. Surgical characteristics

\begin{tabular}{|c|c|c|c|}
\hline Variable & $\begin{array}{l}\text { Standard of Care } \\
\mathrm{N}=139\end{array}$ & $\begin{array}{l}\text { Powered vascular } \\
\text { Stapler } N=131\end{array}$ & $\begin{array}{l}\text { Total } \\
\mathbf{N}=\mathbf{2 7 0}\end{array}$ \\
\hline \multicolumn{4}{|l|}{ Surgery Type, n (\%) } \\
\hline $\begin{array}{l}\text { Laparoscopic } \\
\text { Nephrectomy }\end{array}$ & $112(80.0 \%)$ & $109(83.2 \%)$ & $221(81.9 \%)$ \\
\hline $\begin{array}{l}\text { Laparoscopic } \\
\text { Nephroureterec- } \\
\text { tomy }\end{array}$ & $27(19.4 \%)$ & $22(16.8 \%)$ & $49(18.1 \%$ \\
\hline \multicolumn{4}{|c|}{ Location of affected Kidney, n (\%) } \\
\hline Left & $72(51.8 \%)$ & $73(55.7 \%)$ & $145(53.7 \%)$ \\
\hline Right & $65(46.8 \%)$ & $57(43.5 \%)$ & $122(45.2 \%$ \\
\hline Bilateral & $2(1.4 \%)$ & $1(0.8 \%)$ & $3(1.1 \%)$ \\
\hline \multicolumn{4}{|l|}{$\begin{array}{l}\text { Estimated blood } \\
\text { Loss }(m L)\end{array}$} \\
\hline Mean (SD) & 90.9 (107.4) & $117.6(201.7)$ & $103.9(160.7)$ \\
\hline Median (range) & $50.0(0-900)$ & $50.0(0-1500)$ & $50.0(0-1500)$ \\
\hline \multicolumn{4}{|c|}{$\begin{array}{l}\text { Transfusion of Blood or Blood } \\
\text { Products Required (n, \%) } 3(2.2 \%)\end{array}$} \\
\hline \multicolumn{4}{|c|}{$\begin{array}{l}\text { Procedure Converted to } \\
\text { Open }(\mathrm{n}, \%)\end{array}$} \\
\hline \multicolumn{4}{|c|}{ Procedure Duration (minutes) } \\
\hline Mean (SD) & $153.6(62.2)$ & $157.2(60.0)$ & $155.4(61.1)$ \\
\hline Median (range) & $145.0(48.0,355.0)$ & $145.0(42,381.0)$ & $145.0(42,381.0)$ \\
\hline
\end{tabular}

Table 3. Intraoperative interventions

\begin{tabular}{|c|c|c|c|}
\hline Variable & Standard of Care & $\begin{array}{l}\text { Powered Vascular } \\
\text { Stapler }\end{array}$ & Overall \\
\hline \multicolumn{4}{|c|}{ Vessels Transected, n (\%) ${ }^{a}$} \\
\hline Renal Artery (RA) & $165(53.4 \%)$ & $165(54.1 \%)$ & $330(53.7 \%)$ \\
\hline Renal Vein (RV) & $139(45.0 \%)$ & $133(43.6 \%)$ & $272(44.3 \%)$ \\
\hline $\begin{array}{l}\text { Multiple Vessels } \\
\text { (en bloc) }\end{array}$ & $5(1.6 \%)$ & $7(2.3 \%)$ & $12(2.0 \%)$ \\
\hline \multicolumn{4}{|c|}{$\begin{array}{l}\text { Number of Transections Requiring } \\
\text { Hemostasis Intervention }\end{array}$} \\
\hline $\mathrm{n} /$ total vessels $(\%)$ & $42 / 309(13.6 \%)$ & $56 / 305(18.4 \%)$ & $98 / 614(16.0 \%)$ \\
\hline $\begin{array}{l}95 \% \text { Confidence } \\
\text { Interval }\end{array}$ & $9.8 \%, 17.4 \%$ & $14.0 \%, 22.7 \%$ & $13.1 \%, 18.9 \%$ \\
\hline \multicolumn{3}{|c|}{ Difference, \% (One-sided 95\% CI Upper Bound) } & $4.8 \%(9.6 \%)$ \\
\hline \multicolumn{4}{|c|}{ Types of Intervention, $\mathbf{n}(\%)^{b}$} \\
\hline $\begin{array}{l}\text { Additional } \\
\text { Stapling }\end{array}$ & $7(14.6 \%)$ & $9(14.1 \%)$ & $16(14.3 \%)$ \\
\hline Compression & $1(2.1 \%)$ & $2(3.1 \%)$ & $3(2.7 \%)$ \\
\hline Use of Suture & $1(2.1 \%)$ & 0 & $1(0.9 \%)$ \\
\hline Sealant & $2(4.2 \%)$ & $1(1.6 \%)$ & $3(2.7 \%)$ \\
\hline Use of Energy & $3(6.3 \%)$ & $5(7.8 \%)$ & $8(1.3 \%)$ \\
\hline Clip Placement & $31(64.6 \%)$ & $44(68.8 \%)$ & $75(67.0 \%)$ \\
\hline Other $^{\mathrm{c}}$ & $2(4.2 \%)$ & $3(4.7 \%)$ & $5(4.5 \%)$ \\
\hline $\begin{array}{l}\text { Transfusion and } \\
\text { Additional Procedure }\end{array}$ & $1(2.1 \%)$ & 0 & $1(0.9 \%)$ \\
\hline \multicolumn{4}{|c|}{$\begin{array}{l}{ }^{a} \text { Percentages are calculated using the total number of vessels transected in each group a } \\
\text { the denominator. }\end{array}$} \\
\hline \multicolumn{4}{|c|}{$\begin{array}{l}\text { bMultiple interventions may have been performed on the same firing and therefore the } \\
\text { sum of interventions exceeds the number of transections that required intervention. }\end{array}$} \\
\hline \multicolumn{4}{|c|}{$\begin{array}{l}\text { 'Other Interventions: SOC- Surgicel (2) PVS - Surgicel (1), Surgiflow (1), Pedicle } \\
\text { Clamp (1). }\end{array}$} \\
\hline
\end{tabular}

Table 4. Post-operative interventions related to RA or RV bleeding

\begin{tabular}{|c|c|c|}
\hline Variable & $\begin{array}{l}\text { Standard of Care } \\
n=139\end{array}$ & $\begin{array}{l}\text { Powered Vascular } \\
\text { Stapler } n=13\end{array}$ \\
\hline $\begin{array}{l}\text { Subjects Requiring Post-Operative } \\
\text { Intervention, } \mathrm{n} / \mathrm{N}(\%)\end{array}$ & $2 / 139(1.4 \%)$ & $1 / 131(0.8 \%)$ \\
\hline \multicolumn{3}{|l|}{ Types of Intervention, $\mathrm{n}$} \\
\hline Transfusion & 2 & 1 \\
\hline Additional Procedure required & 1 & 0 \\
\hline
\end{tabular}

procedure-related AEs were also classified for clinical relevance per the Clavien-Dindo surgical complications scale ${ }^{13}$. There were a total of 160 procedure-related AEs reported during the study with no meaningful differences observed between treatment groups in the distribution of Clavien-Dindo grades: 108 (67.5\%) were scored as grade 1, $46(28.8 \%)$ were grade $2,5(3.1 \%)$ were grade 3 , and $1(0.6 \%)$ was grade 4 . Among the seven device-related AEs, two were grade 1 , three were grade 2 , and two were grade 3 .

\section{Surgeon questionnaires}

Additional data were collected by asking the surgeons to complete a satisfaction questionnaire and Surgery task load index (SURG-TLX) for the PVS and SOC devices. Similar results were observed between PVS and SOC groups on the Surgery Task Load Index for overall mean scores, as well as each of the six subscale scores (data not shown). Surgeon satisfaction with device usability was also similar between groups with $82.8 \%$ of SOC cases being rated as satisfied or very satisfied compared to $80.1 \%$ in the PVS group.

\section{Discussion}

In this randomized study, a higher percentage of transections requiring intraoperative haemostatic intervention was observed in 
the PVS group (18.4\%) compared to the SOC subjects (13.6\%). The difference in haemostatic intervention rates between treatment groups was not statistically significant, though the upper bound of the $95 \%$ confidence interval for the difference between PVS and SOC did exceed a pre-defined performance criterion of $3 \%$. An expected intervention rate of $6 \%$ was used for determination of the study sample size, however the observed intervention rate of $13.6 \%$ in the control (SOC) arm suggests that $3 \%$ as the upper limit for the difference between PVS and SOC may have been too restrictive for the procedures in this study. Thus, while the given sample sizes were expected to provide reasonable precision for estimation of the haemostasis intervention rates, the study was underpowered for demonstrating that the difference between treatment group comparison could achieve the $3 \%$ criterion. The relatively high intervention rates observed in both treatment groups could reflect the number of complex cases. Only subjects undergoing total nephrectomies (simple or radical), or nephroureterectomy, were included in this study. In addition, 76 of 183 (41.5\%) pathologically assessed kidney tumours were stage IIIA malignancy or higher, suggesting a substantial proportion of more complex cases, though the distribution of tumour stages was similar between treatment groups.

The types of additional intraoperative haemostatic interventions were consistent with those expected in these types of surgeries and the higher rate in the PVS group could be representative of surgeons who had not used the device regularly or consistently prior to participation in the study. This is likely reflected in the higher utilization of clip placement within the PVS group, as clip placement is the most common intervention used in these surgeries and there may have been a reliance on this common intervention for greater assurance of haemostatic security as surgeons adapted to a new technology. It should be noted that a minimum of two training cases were performed by surgeons prior to participating in the study. The associated findings of no clinically meaningful differences between SOC and PVS in blood loss, requirement for transfusion, or need for postoperative interventions related to bleeding attest to the point that there was no haemostatic impact with the new device.

The frequencies of specific AEs were similar between the device groups. Of 160 procedure-related AEs, 154 (96.3\%) were scored as Clavien-Dindo grade 1 or grade 2. Procedure-related AEs with higher Clavien-Dindo grades $(\geq 3)$ occurred in 6 of $270(2.2 \%)$ total procedures, including 3 subjects from each treatment group. These consisted of a colon injury, post-operative haemorrhage, and wound infection in the SOC group, and hypoxia, procedural haemorrhage, and an arteriovenous fistula complication in PVS subjects. These findings were similar to a large British Association of Urological Surgeons (BAUS) dataset [14], in which complications with ClavienDindo grades $\geq 3$ were noted in $3.9 \%$ of 6,042 nephrectomies. This dataset included both laparoscopic and open nephrectomies as well as nephroureterectomies [14].

Given that for all surgeons participating in this study the PVS device was not the current standard of care, it is encouraging that a high degree of satisfaction with device usability was reported for the PVS $(80.1 \%)$, which was similar to satisfaction levels for the SOC staplers $(82.8 \%)$. Moreover, considering the types and frequencies of intraoperative haemostatic interventions, the severity of those interventions, and supporting surgeon questionnaire results, the PVS exhibited equivalent performance and clinical utility compared to SOC, for sealing renal arteries and renal veins.

\section{Funding}

Funding for this study has been provided by Ethicon Endo-Surgery, Inc.

\section{Disclosure Statement}

EEC, MLS, JRW, DWS and EJF are employed by Ethicon EndoSurgery.

\section{Ethical Approval}

All procedures performed in studies involving human participants were in accordance with the ethical standards of the institutional and/ or national research committee and with the 1964 Helsinki declaration and its later amendments or comparable ethical standards.

\section{References}

1. Beisland C, Medby PC, Sander S, Beisland HO (2000) Nephrectomy - indications, complications and postoperative mortality in 646 consecutive patients. Eur Urol 37: 58-64. [Crossref]

2. Rafique M (2007) Nephrectomy: indications, complications and mortality in 154 consecutive patients. J Pak Med Assoc 57: 308-11. [Crossref]

3. Srirangam SJ, van Cleynenbreugel B, van Poppel H (2009) Laparoscopic nephroureterectomy: the distal ureteral dilemma. Adv Urol. [Crossref]

4. Johal NS, Kraklau D, Cuckow PM (2005) The role of unilateral nephrectomy in the treatment of nephrogenic hypertension in children. BJU Int 95: 140-142. [Crossref]

5. Raber B, Westmoreland M, Arnold D, Derek B2, Lueking R, et al. (2017) Laparoscopic donor nephrectomy: A single institution minimally invasive general surgeon experience 1999-2013. Am J Surg 214:1220-1225. [Crossref]

6. Rampersad C, Patel P, Koulack J, McGregor T (2016) Back-to-back comparison of mini-open vs. laparoscopic technique for living kidney donation. Can Urol Assoc J 10: 253-257. [Crossref]

7. McGregor TB, Patel P, Sener A, Chan G (2017) Vascular control during laparoscopic kidney donation. Can J Surg 60: 150-1. [Crossref]

8. Cacciamani GE, Medina LG, Gill T, Abreu A, Sotelo R, et al. (2018) Impact of Surgical Factors on Robotic Partial Nephrectomy Outcomes: Comprehensive Systematic Review and Meta-Analysis. J Urol 200: 258-274. [Crossref]

9. Hamilton ZA, Uzzo RG, Larcher A, Lane BR, Ristau B, et al. (2018) Comparison of functional outcomes of robotic and open partial nephrectomy in patients with pre-existing chronic kidney disease: a multicenter study. World J Urol 36: 1255-62. [Crossref]

10. Kuroda H, Yoshida T, Sakao Y (2017) A powered vascular staple for the application of segmental bronchial closure in thoracoscopic anatomic segmentectomy. J Thorac Dis 9: 5352-5354. [Crossref]

11. Ng CS, Pickens A, Siegel JM, Clymer JW, Cummings JF (2016) A novel narrow profile articulating powered vascular stapler provides superior access and haemostasis equivalent to conventional devices. Eur J Cardiothorac Surg 49i73-8. [Crossref]

12. Miller DL, Roy S, Kassis ES, Yadalam S, Ramisetti S, et al. (2018) Impact of Powered and Tissue-Specific Endoscopic Stapling Technology on Clinical and Economic Outcomes of Video-Assisted Thoracic Surgery Lobectomy Procedures: A Retrospective, Observational Study. Adv Ther 35: 707-23. [Crossref]

13. Dindo D, Demartines N, Clavien PA (2004) Classification of surgical complications: a new proposal with evaluation in a cohort of 6336 patients and results of a survey. Ann Surg 240: 205-213. [Crossref]

14. Henderson JM, Fowler S, Joyce A, Dickinson A, Keeley FX; BAUS (2015) Perioperative outcomes of 6042 nephrectomies in 2012: surgeon-reported results in the UK from the British Association of Urological Surgeons (BAUS) nephrectomy database. BJU Int 115: 121-126. [Crossref]

Copyright: (C)2019 Master VA. This is an open-access article distributed under the terms of the Creative Commons Attribution License, which permits unrestricted use, distribution, and reproduction in any medium, provided the original author and source are credited. 